Copyright (C 2014 IEEE. Personal use of this material is permitted. Permission from IEEE must be obtained for all other uses, in any current or future media, including reprinting/republishing this material for advertising or promotional purposes, creating new collective works, for resale or redistribution to servers or lists, or reuse of any copyrighted component of this work in other works. 


\title{
Secondary Control in Microgrids for Dynamic Power Sharing and Voltage/Frequency Adjustment
}

\author{
Ruwan P.S. Chandrasena, Farhad Shahnia, Arindam Ghosh and Sumedha Rajakaruna \\ Electrical and Computer Engineering Department \\ Curtin University \\ Perth, Australia \\ ruwan.chandrasena@curtin.edu.au
}

\begin{abstract}
This paper discusses the operation of the central controller for a microgrid. The central controller is responsible to maintain the voltage and frequency of the microgrid within the acceptable ranges while the distributed energy resources supply the required power demand of the loads based on the droop control. The desired ratio among the output active power of the distributed energy resources is determined by the distribution network tertiary controller. To achieve a dynamic variation for the output active power ratios among the distributed energy resources, the central controller has to adjust the references of the rated active power and the droop control coefficients. As this may lead to unacceptable voltage and frequency in the microgrid, the central controller has to adjust the references of the voltage and frequency consequently. The proposed central controller strategy for the microgrid is validated through PSCAD/EMTDC simulations.
\end{abstract}

Index Terms-Microgrid, Distributed Energy Resource (DER), Power Sharing, Secondary Controller.

\section{INTRODUCTION}

The ever increasing energy demand, along with the necessity of cost reduction and higher reliability requirements, are driving the modern power systems towards using distributed energy resources (DER) as an alternative to the expansion of the current electricity distribution networks [1-2].

Microgrids (MG) are systems with clusters of DERs and loads. To deliver high quality and reliable power, the MG should appear as a single controllable unit that responds to changes in the system [3]. In MGs, DERs are connected to the network through coupling inductances and are controlled to deliver the desired active and reactive power to the system. The power sharing among the DERs can be achieved by controlling two independent quantities, frequency and fundamental voltage magnitude, of the DERs based on droop control [4-7].

General introduction on MG basics, including the architecture, protection and power management is given in [8-9]. A review of ongoing research projects on MG in US, Canada, Europe and Japan is presented in [9-10]. Different power management strategies and controlling algorithms for a $\mathrm{MG}$ are proposed in [11-14]. Reference [15] has evaluated the feasibility of MGs operation during islanding and synchronisation periods.

A three-level hierarchical control architecture can be identified for MG control as presented in [10]. The distribution network tertiary controller analyses the data such as load/ weather forecast, electricity market and economic dispatch to define the references for the output power of each DER within the MG. These references are communicated to the MG secondary (central) controller which passes this information to the primary level controllers. The primary controllers are within each DER and are responsible for controlling the DER outputs based on the references received from the central controller. Hence, the required output of a DER can vary from zero to its maximum capacity based on the commands of the tertiary controller.

To realize a power ratio among the DERs in the MG, droop controller coefficients need to be designed properly [6]. Many researches propose to design the coupling inductances of the DERs such that their ratio becomes reciprocal to the desired ratio of the output powers of the DERs. However, it is not practical to adjust the coupling inductances dynamically for varying output power ratios. A virtual impedance-based technique is introduced and utilized in [16] to overcome this problem. However, in general, dynamic power sharing ratios can be achieved by adjusting the droop control coefficients by the help of the central controller for any ratios of the coupling inductances. However, the central controller should also monitor and adjust the references for the voltage and frequency within the MG if they are fallen outside acceptable ranges.

In this paper, the operation, control and power sharing among parallel converter-interfaced DERs, operating in voltage control mode, are investigated for autonomous mode. Through the extensive simulation results carried out by PSCAD/EMTDC, it is demonstrated that the output power ratio among the available DERs in the MG can change dynamically by the help of the central controller. In addition, it is demonstrated that the central controller can manage to keep the voltage and frequency within acceptable ranges by dynamically adjusting the references following such violations.

\section{MICROGRID STRUCTURE AND CONTROL}

Let us consider the fundamental MG structure as shown in Fig. 1(a). The considered MG system consists of two converter-interfaced DERs. DERs such as photovoltaic cells (PV), fuel cells and batteries are usually connected to the MG through voltage source converters (VSC) and a properly tuned filter. For simplicity, in the rest of the paper, each DER 
along with its VSC and filter structure is simply called DER system, as shown in Fig. 1(a). DER systems are connected through coupling inductances $\left(L_{T}\right)$ to the feeder and are controlled to supply the load requirements within the MG. Although the loads can be distributed throughout the $\mathrm{MG}$, a centralized load is shown in Fig. 1(a). It is to be noted that the considered DER systems in this paper are working in voltage control mode and their instantaneous output power, are within their rated capacities.

\section{A. MG Hierarchical Control System}

For proper operation of a MG within a distribution network, a three-level hierarchical control system is presented and discussed in [10], as shown in Fig. 1(a). This hierarchical control system is discussed below:

- The primary controller is responsible for appropriate switchings in the converters of the DERs such that the desired output power is observed at the output of the DER converters. This controller is composed of two control loops- the outer-loop control which is responsible for proper output power control of DERs in the MG and the innerloop control which is responsible for proper tracking of the generated references (by the outer-loop control) at the output of the DER converters. The primary control of the DERs, based on voltage-control strategy, is presented in [10]. This controller is not the focus of this paper.

- The secondary controller is the central controller of the MG. This controller sends the desired (reference) output power to each DER in the system. In grid-connected mode, the desired output power of each DER converter is received from the tertiary controller. However, in autonomous mode, this controller sends reference signals to DER converters in the form of voltage magnitude and angle, based on monitoring the network voltage and frequency, whenever required. This controller runs in a slower time frame compared to that of the primary control [17]. The secondary control is the main scope of this research and is discussed in rest of the paper.

- The tertiary controller communicates with the central controllers in the MG. In general, this controller can utilize load/weather forecast, electricity market, economic dispatch and unit commitment information for optimal power flow of the network and MGs [18]. The tertiary controller is not the focus of this paper and is not discussed in this paper.

\section{B. DER Converter Structure}

The DERs are assumed to be connected to the MG through VSCs. The VSC structure consists of three single-phase Hbridges, using Insulated Gate Bipolar Transistors (IGBT), as shown in Fig. 1(b). Each IGBT has anti-parallel diode and snubber circuits. The outputs of each H-bridge are connected to a single-phase transformer, with $1: a$ ratio, and three secondary windings of the transformers are star-connected. These transformers provide galvanic isolation as well as voltage boosting capability if required. As the converters are desired to be voltage-controlled, an LC filter is utilized for each phase at the outputs of the converter. In this figure, the (a)
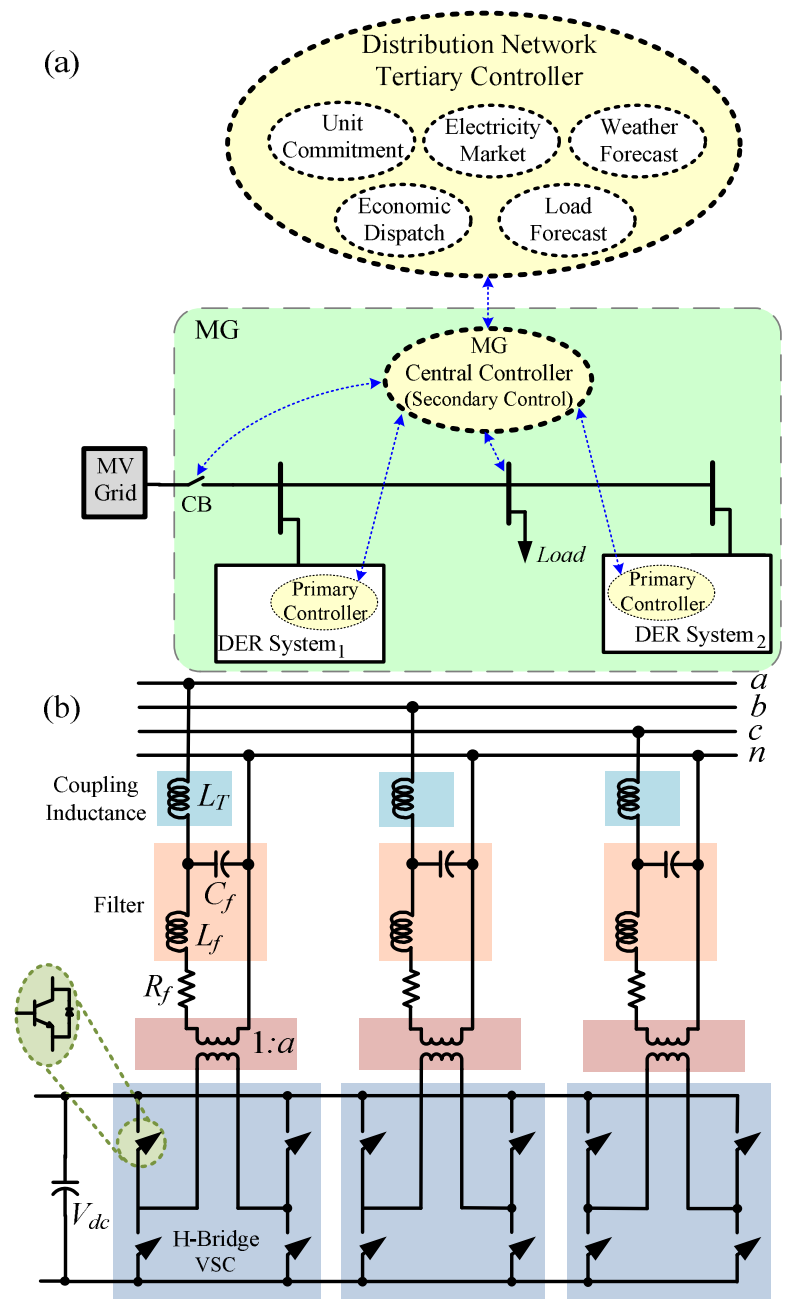

Fig. 1. (a) Schematic diagram of the MG network under consideration along with its hierarchical control system, (b) The VSC and filter structure of the DER system.

resistance $R_{f}$ represents the switching and transformer core losses. The filter inductance $L_{f}$ and the filter capacitor $C_{f}$ are designed to suppress the switching harmonics from the current and voltages.

\section{Droop Control}

The active power $(p)$ and reactive power $(q)$ supplied by the DER systems to the MG through the coupling inductance $\left(L_{T}\right)$ are given by

$$
\begin{aligned}
& p=\frac{\left|V_{i}\right| \times\left|V_{j}\right|}{\omega L_{T}} \sin \delta_{i j} \\
& q=\frac{\left.\left|V_{i}\right| V_{j}\left|\cos \delta_{i j}-\right| V_{i}\right|^{2}}{\omega L_{T}}
\end{aligned}
$$

where $V_{i}$ and $V_{j}$ are respectively the MG side and filter side voltages at two terminals of the coupling inductance $\left(L_{T}\right), \delta_{i j}$ is the angle difference between these two voltages and $V=$ $|V| \angle \delta$ is the phasor representation of $v(t)$. The average active power $(P)$ and reactive power $(Q)$ can then be calculated by passing $p$ and $q$ through Low Pass Filters (LPF). 
In autonomous mode, it is assumed that the frequency of the MG reduces by $\Delta \omega$, as the DER system increases its output active power from zero to the rated value. The slop of this variation is referred to as $m$ and is calculated from

$m=\frac{\Delta \omega}{P_{\text {rated }}}=\frac{\omega_{\text {max }}-\omega_{\text {rated }}}{P_{\text {rated }}}=2 \pi \frac{f_{\text {max }}-f_{\text {rated }}}{P_{\text {rated }}}$

where $f_{\text {rated }}$ and $f_{\max }$ are assumed to be respectively 50 and 51 $\mathrm{Hz}$ and $m>0$. Hence, $P_{\text {rated }}$ is the output active power form the DER system when the frequency of the $\mathrm{MG}$ is equal to $f_{\text {rated. }}$.

Assuming for two DERs with different ratings, $\Delta \omega$ is constant (i.e. $\Delta \omega_{1}=\Delta \omega_{2}$ ), the ratio of $P-f$ droop coefficient between the two DER systems is

$m_{1} P_{\text {rated,1 }}=m_{2} P_{\text {rated }, 2} \Rightarrow \frac{m_{1}}{m_{2}}=\frac{P_{\text {rated }, 2}}{P_{\text {rated, } 1}}$

Similarly, it is assumed that the voltage of the MG reduces by $\Delta V$, when the DER system increases its output reactive power from zero to the rated value. The slop of this variation is referred to as $n$ and is calculated from

$n=\frac{\Delta V}{Q_{\text {rated }}}=\frac{V_{\text {max }}-V_{\text {rated }}}{Q_{\text {rated }}}$

where $n>0$. In this study, $V_{\text {rated }}$ is assumed to be $1 \mathrm{pu}$ while $V_{\max }$ is assumed to be $1.1 \mathrm{pu}$. It is to be noted that $V_{\text {rated }}$ is the desired voltage in the MG. Hence, $Q_{\text {rated }}$ is the output reactive power from the DER system when the voltage magnitude of the MG is equal to $V_{\text {rated }}$.

Assuming for two DERs with different ratings, $\Delta V$ is constant (i.e. $\Delta V_{1}=\Delta V_{2}$ ), the ratio of $Q-V$ droop coefficient between two DER systems is

$n_{1} Q_{\text {rated }, 1}=n_{2} Q_{\text {rated }, 2} \Rightarrow \frac{n_{1}}{n_{2}}=\frac{Q_{\text {rated }, 2}}{Q_{\text {rated }, 1}}$

Decentralized power sharing among two DER systems in the MG can be achieved by changing the voltage magnitude and angle of DER systems using the droop control as

$f_{j}=f_{\text {rated }}+m\left[\frac{X_{\text {line }}}{Z_{\text {line }}}\left(P_{\text {rated }}-P\right)-\frac{R_{\text {line }}}{Z_{\text {line }}}\left(Q_{\text {rated }}-Q\right)\right]$

$\left|V_{j}\right|=V_{\text {rated }}+n\left[\frac{R_{\text {line }}}{Z_{\text {line }}}\left(P_{\text {rated }}-P\right)+\frac{X_{\text {line }}}{Z_{\text {line }}}\left(Q_{\text {rated }}-Q\right)\right]$

where $Z_{\text {line }}=R_{\text {line }}+j X_{\text {line }}$ is the equivalent impedance of the $\mathrm{MG}$ feeder between the DER and the load. If the MG feeder lines are assumed to have a higher inductive characteristic (i.e. $Z_{\text {line }} \approx j X_{\text {line }}$, , the active and reactive powers are assumed to be decoupled. Hence, (6) is simplified further as

$$
\begin{gathered}
f_{j}=f_{\text {rated }}+m\left(P_{\text {rated }}-P\right) \\
\left|V_{j}\right|=V_{\text {rated }}+n\left(Q_{\text {rated }}-Q\right)
\end{gathered}
$$

For two DER systems, $V_{\text {rated }}$ and $\delta_{\text {rated }}$ are the same for both of the DER systems while $m, n, P_{\text {rated }}$ and $Q_{\text {rated }}$ are as described in (2)-(4), from Fig. 1(a), it can be seen that
$\frac{P_{1}}{P_{2}}=\frac{m_{2}}{m_{1}} \quad, \quad \frac{Q_{1}}{Q_{2}}=\frac{n_{2}}{n_{1}}$

Now, let us consider the MG network of Fig. 1(a) with 2 DERs supplying a common load. DER-1 has a coupling inductance of $L_{T, 1}$ at its output while DER-2 has a coupling inductance of $L_{T, 2}$. DER-1 is connected to the load through a feeder impedance of $Z_{\text {line }, 1} \approx j X_{\text {line }, 1}$ where DER-2 is connected to the load through a feeder impedance of $Z_{\text {line }, 2} \approx j X_{\text {line }, 2}$. Assuming the voltage at load PCC is $V_{\text {load }}$, the active and reactive power supplied from each DER to the load can be expressed as

$$
\begin{aligned}
& p_{1}=\frac{\left|V_{\text {load }}\right|\left|V_{1}\right| \sin \delta_{1}}{\omega L_{T, 1}+\omega L_{\text {line }, 1}} \\
& p_{2}=\frac{\left|V_{\text {load }}\right|\left|V_{2}\right| \sin \delta_{2}}{\omega L_{T, 2}+\omega L_{\text {line }, 2}}
\end{aligned}
$$

where $\left|V_{1}\right|$ and $\left|V_{2}\right|$ are respectively the voltage magnitude at the output of DER-1 and DER-2 and $\delta_{1}$ is the voltage angle difference between the output of DER-1 and the load while $\delta_{2}$ is the voltage angle difference between the output of DER-2 and the load.

Assuming $X_{\text {line }}<\omega L_{T}$, the active/reactive power delivered from the DER system to the load is highly dependent on the coupling inductance at the output of the DER system and is not affected by the MG line reactance. Hence, (9) can be simplified as

$p_{1} \approx \frac{\left|V_{\text {load }}\right|\left|V_{1}\right| \sin \delta_{1}}{\omega L_{T, 1}}$
$p_{2} \approx \frac{\left|V_{\text {load }}\right|\left|V_{2}\right| \sin \delta_{2}}{\omega L_{T, 2}}$

From (10), the ratio of the average active power between these two DER systems can be given as

$\frac{P_{1}}{P_{2}}=\frac{L_{T, 2}}{L_{T, 1}} \frac{\left|V_{1}\right| \sin \delta_{1}}{\left|V_{2}\right| \sin \delta_{2}}$

Now, let us assume the desired active power ratio between these two DER systems is $P_{1} / P_{2}=k_{p}$ where $k_{p}>0$. In many references such as $[3,11]$, for simplicity, it is assumed that

$$
\left\{\begin{array}{l}
\frac{P_{1}}{P_{2}}=k_{p} \\
\frac{L_{T, 2}}{L_{T, 1}}=k=k_{p}
\end{array} \Rightarrow\left|V_{1}\right| \sin \delta_{1}=\left|V_{2}\right| \sin \delta_{2}\right.
$$

The ratio of the reactive power output between two DERs can be calculated from (1) as

$$
\frac{Q_{1}}{Q_{2}}=\frac{L_{T, 2}}{L_{T, 1}} \frac{\left|V_{1}\right| \cos \delta_{1}-\left|V_{3}\right|}{\left|V_{2}\right| \cos \delta_{2}-\left|V_{3}\right|}
$$


Now, let us assume that the reactive power ratio between these two DER systems is $Q_{1} / Q_{2}=k_{q}$ where $k_{q}>0$. In many references such as $[3,11]$, for simplicity, it is assumed that

$$
\left\{\begin{array}{l}
\frac{Q_{1}}{Q_{2}}=k_{q}=k_{p} \\
\frac{L_{T, 2}}{L_{T, 1}}=k=k_{q}
\end{array} \quad \Rightarrow\left|V_{1}\right| \cos \delta_{1}=\left|V_{2}\right| \cos \delta_{2}\right.
$$

However, in general, it can be assumed that coupling inductances of the two DER systems can have a ratio of $k^{\prime}$ where $k^{\prime}>0$. In such a case, we have

$$
\left\{\begin{array}{l}
\frac{P_{1}}{P_{2}}=k_{p} \\
\frac{L_{T, 2}}{L_{T, 1}}=k^{\prime}
\end{array} \Rightarrow\left|V_{1}\right| \sin \delta_{1}=\frac{k_{p}}{k^{\prime}}\left|V_{2}\right| \sin \delta_{2}=k^{\prime \prime}\left|V_{2}\right| \sin \delta_{2}\right.
$$

where $k^{\prime \prime}=k_{p} / k^{\prime}$. From (15), it can be seen that under such conditions, the voltages at the outputs of both of the DER systems are correlated by a factor of $k^{\prime \prime}$. It is to be noted that under such condition, the ratio among the reactive powers of the two DER systems will be as

$$
\left\{\begin{array}{l}
\frac{Q_{1}}{Q_{2}}=k_{q} \\
\frac{L_{T, 2}}{L_{T, 1}}=k^{\prime}
\end{array} \Rightarrow\left|V_{1}\right| \cos \delta_{1}-\left|V_{3}\right|=\frac{k_{q}}{k^{\prime}}\left(\left|V_{2}\right| \cos \delta_{2}-\left|V_{3}\right|\right)\right.
$$

Hence, from (15) and (16), it can be seen that in general $k_{p} \neq$ $k_{q}$. Therefore, the output active power of the two DER systems can be controlled to be equal to the desired value of $k_{p}$ while the ratio of the reactive power outputs among these two DER systems will not be equal to $k_{p}$. Hence, if only active power ratio is going to be changed dynamically, any ratio of the coupling inductances can be selected (considering the system stability). If in addition to the active power, the reactive power ratio is also desired to change dynamically, a virtual impedance technique as discussed in [16] should be utilized.

It is desired that the voltage angle difference on two sides of the coupling inductance (i.e. $\delta_{i j}$ ) to be a small value so that it is on the linear section of sinusoidal $P-\delta$ characteristic of (1). Similarly, it is desired that the voltage drop across the coupling inductance (i.e. $\left|V_{1}\right|-\left|V_{\text {load }}\right|$ ) to be small (i.e. 1-2 $\%)$.

\section{Microgrid Secondary Control}

The secondary level control (central controller) communicated with the distribution network tertiary controller as well as the primary controller of all DER systems within the MG. The main responsibility of the central controller is to provide the references and set points, required for the primary controller of each DER system. These set points are later used to define the output active and reactive power of each DER system. This is valid for both grid-connected and autonomous modes. It is to be noted that the central controller does not operate instantaneously and has larger time steps $(\Delta T)$ com- pared to the primary controller of each DER system. Hence, it runs in a slower time frame compared to the primary controllers; however, it should be faster than the operation time of the under/overvoltage and under/over frequency relays within the MG.

In grid-connected mode, each DER generates an output power based on its MPPT or the level defined by the distribution network tertiary controller. Hence, in this case, the MG central controller receives the desired ratio of the output power of the DER systems from the tertiary controller and passes those information (set points) to each DER system.

In autonomous mode, each DER has to generate a portion of the load requirements within the MG. This portion is again defined by the tertiary controller and passed to each DER system through the central controller. However, in this case, as the voltage and frequency in the MG is not dictated by the grid, the central controller is also responsible for monitoring these two parameters. According to the load variations in the MG, the output power of each DER will vary; however, if the voltage and frequency of the MG increases above or drops below the acceptable thresholds, the central controller defines new settings for the primary controller of each DER system and then sends these set points to them. It is to be noted that this procedure should take place faster than the pre-defined operation time for under/over frequency relays in the MG.

The central controller consists of two modules, namely dynamic power ratio adjustment module and droop curve adjustment module, as described in detail, below.

\section{A. Dynamic Power Ratio Adjustment Module}

In grid-connected as well as the autonomous mode, the distribution network tertiary controller defines the desired output power ratio among the DERs within a MG. Hence, the ratio among the output powers of two DERs can change dynamically based on the commands from the tertiary controller which are transferred to the DER systems through the MG central controller. Let us assume that at $t=t_{1}$, the ratio of the output active power of two DERs (i.e. DER-1 and DER-2 of Fig. 1a) is $k_{p 1}$. Hence, from (8), we have $P_{\text {rated, } 2} / P_{\text {rated, } 1}=m_{1} / m_{2}$ $=k_{p 1}$. In this case, from (7), we have

$f_{1}^{t_{1}}=f_{\text {rated }}+m_{1}\left(P_{\text {rated, } 1}-P_{1}^{t_{1}}\right)$

The tertiary controller decides to change this ratio to $k_{p 2}$ at $t=$ $t_{2}$. Hence, from (7), we have

$f_{1}^{t_{2}}=f_{\text {rated }}+m_{1}\left(P_{\text {rated, } 1}-P_{1}^{t_{2}}\right)$

Since the load demand in $t_{1}$ and $t_{2}$ is same, the total active power generation by the DERs will be the same at these two time intervals. Hence, we have

$P_{1}^{t_{1}}+P_{2}^{t_{1}}=P_{1}^{t_{2}}+P_{2}^{t_{2}}$

Replacing the power ratios in (19), it can be simplified as

$P_{1}^{t_{1}}+k_{p 1} P_{1}^{t_{1}}=P_{1}^{t_{2}}+k_{p 2} P_{1}^{t_{2}} \Rightarrow P_{1}^{t_{1}}\left(1+k_{p 1}\right)=P_{1}^{t_{2}}\left(1+k_{p 2}\right)$

$\Rightarrow P_{1}^{t_{2}}=P_{1}^{t_{1}} \frac{1+k_{p 1}}{1+k_{p 2}}$ 
From (17),(18) and (20), the frequency variation due to this ratio change is calculated as

$$
\begin{aligned}
\Delta f^{t} & =f_{1}^{t_{2}}-f_{1}^{t_{1}}=m_{1}\left(P_{1}^{t_{1}}-P_{1}^{t_{2}}\right)=P_{1}^{t_{1}} m_{1}\left(1-\frac{1+k_{p 1}}{1+k_{p 2}}\right) \\
& =\frac{k_{p 2}-k_{p 1}}{1+k_{p 2}} m_{1} P_{1}^{t_{1}}
\end{aligned}
$$

It is to be noted that this frequency variation is acceptable as far as the new frequency of the $\mathrm{MG}$ is between $f_{\max }$ and $f_{\min }$. Therefore, we have

$$
\begin{aligned}
& f_{\min } \leq f_{1}^{t_{1}}+\Delta f^{t} \leq f_{\max } \Rightarrow f_{\min }-f_{1}^{t_{1}} \leq \Delta f^{t} \leq f_{\max }-f_{1}^{t_{1}} \\
& f_{\min }-f_{1}^{t_{1}} \leq \frac{k_{p 2}-k_{p 1}}{1+k_{p 2}} m_{1} P_{1}^{t_{1}} \leq f_{\max }-f_{1}^{t_{1}}
\end{aligned}
$$

Assuming

$$
H_{1}=\frac{f_{\min }-f_{1}^{t_{1}}}{m_{1} P_{1}^{t_{1}}} \text { and } H_{2}=\frac{f_{\max }-f_{1}^{t_{1}}}{m_{1} P_{1}^{t_{1}}}
$$

to prevent non-acceptable frequency in the $\mathrm{MG}, k_{p 2}$ must fulfil the following constraints.

$$
\left\{\begin{array}{l}
k_{p 2}>0 \\
k_{p 2} \leq\left(k_{p 1}+H_{1}\right) /\left(1-H_{1}\right) \\
k_{p 2} \leq\left(k_{p 1}+H_{2}\right) /\left(1-H_{2}\right)
\end{array}\right.
$$

The similar study can be repeated for the voltage magnitude in the MG. Assuming at $t=t_{1}$, the ratio of the output reactive power of DER-1 and DER-2 of Fig. 1a is $k_{q 1}$, from (8), we have $Q_{\text {rated,2 }} / Q_{\text {rated,1 }}=n_{1} / n_{2}=k_{q 1}$. In this case, from (7), we have

$\left|V_{1}\right|^{t_{1}}=V_{\text {rated }}+n_{1}\left(Q_{\text {rated }, 1}-Q_{1}^{t_{1}}\right)$

The tertiary controller decides to change this ratio to $k_{q 2}$ at $t=$ $t_{2}$. Hence, from (7), we have

$\left|V_{1}\right|^{t_{2}}=V_{\text {rated }}+n_{1}\left(Q_{\text {rated, } 1}-Q_{1}^{t_{2}}\right)$

Since the load demand in $t_{1}$ and $t_{2}$ is same, the total reactive power generation by the DERs will be the same at these two time intervals. Hence, we have

$Q_{1}^{t_{1}}+Q_{2}^{t_{1}}=Q_{1}^{t_{2}}+Q_{2}^{t_{2}}$

Replacing the power ratios in (26), it can be simplified as

$$
\begin{aligned}
& Q_{1}^{t_{1}}+k_{q 1} Q_{1}^{t_{1}}=Q_{1}^{t_{2}}+k_{q 2} Q_{1}^{t_{2}} \Rightarrow Q_{1}^{t_{1}}\left(1+k_{q 1}\right)=Q_{1}^{t_{2}}\left(1+k_{q 2}\right) \\
& \Rightarrow Q_{1}^{t_{2}}=Q_{1}^{t_{1}} \frac{1+k_{q 1}}{1+k_{q 2}}
\end{aligned}
$$

From (24), (25) and (27), the voltage variation due to this ratio change is calculated as

$$
\begin{aligned}
\Delta\left|V_{1}\right|^{t} & =\left|V_{1}\right|^{t_{2}}-\left|V_{1}\right|^{t_{1}}=n_{1}\left(Q_{1}^{t_{1}}-Q_{1}^{t_{2}}\right)=Q_{1}^{t_{1}} n_{1}\left(1-\frac{1+k_{q 1}}{1+k_{q 2}}\right) \\
& =\frac{k_{q 2}-k_{q 1}}{1+k_{q 2}} n_{1} Q_{1}^{t_{1}}
\end{aligned}
$$

It is to be noted that this voltage magnitude variation is acceptable as far as the new voltage magnitude of the $\mathrm{MG}$ is between $|V|_{\max }$ and $|V|_{\min }$. Therefore, we have

$$
\begin{aligned}
& |V|_{\text {min }} \leq\left|V_{1}\right|^{t_{1}}+\Delta|V|^{t} \leq|V|_{\max } \\
& \Rightarrow|V|_{\text {min }}-\left|V_{1}\right|^{t_{1}} \leq \Delta|V|^{t} \leq|V|_{\max }-\left|V_{1}\right|^{t_{1}} \\
& \Rightarrow|V|_{\text {min }}-\left|V_{1}\right|^{t_{1}} \leq \frac{k_{q 2}-k_{q 1}}{1+k_{q 2}} n_{1} Q_{1}^{t_{1}} \leq|V|_{\max }-\left|V_{1}\right|^{t_{1}}
\end{aligned}
$$

Assuming

$$
G_{1}=\frac{|V|_{\min }-\left|V_{1}\right|^{t_{1}}}{n_{1} Q_{1}^{t_{1}}} \text { and } G_{2}=\frac{|V|_{\max }-\left|V_{1}\right|^{t_{1}}}{n_{1} Q_{1}^{t_{1}}}
$$

to prevent non-acceptable voltage magnitude in the $\mathrm{MG}, k_{q 2}$ must fulfil the following constraints.

$$
\left\{\begin{array}{l}
k_{q 2}>0 \\
k_{q 2} \leq\left(k_{q 1}+G_{1}\right) /\left(1-G_{1}\right) \\
k_{q 2} \leq\left(k_{q 1}+G_{2}\right) /\left(1-G_{2}\right)
\end{array}\right.
$$

If $k_{p 2}$ causes violations in the $\mathrm{MG}$ frequency and voltage magnitude, the $f_{\text {rated }}$ and $V_{\text {rated }}$ parameters for the DER systems should be adjusted properly to recover the voltage magnitude and frequency to the acceptable ranges, as discussed below. Fig. 2(b) shows schematically the dynamic power ratio adjustment principle.

\section{B. Droop Curve Adjustment Module}

In grid-connected mode, the voltage and frequency of the MG is dictated by the grid. However, in autonomous mode, these parameters are regulated indirectly by the output active and reactive power of the DER systems. Hence, the droop curve adjustment module is only active when the $\mathrm{MG}$ is in autonomous mode. In this case, if the voltage magnitude or frequency within the MG rises above or drops below the acceptable thresholds, the central controller takes action to shift up or down the rated values of the droop characteristic. It is desired that for the same output powers of the DER system, the MG voltage magnitude and frequency is retained close to the desired values. For this, the difference between the frequency of the MG with its desired value should be calculated. Let us call this difference as $\Delta \omega$. To adjust the MG frequency to the desired value, a new rated frequency can be defined by adding the previous (old) rated frequency by $\Delta \omega$ as

$$
f_{\text {rated }}^{\text {new }}=f_{\text {rated }}^{\text {old }}+\Delta f=f_{\text {rated }}^{\text {old }}+\left(f_{\text {desired }}-f\right)
$$

Similarly, the difference between the voltage magnitude of the $\mathrm{MG}$ with its desired value should be calculated. Let us call this difference as $\Delta V$. To adjust the MG voltage magnitude to the desired value, a new rated voltage can be defined by adding the previous (old) rated voltage magnitude by $\Delta V$ as

$V_{\text {rated }}^{\text {new }}=V_{\text {rated }}^{\text {old }}+\Delta V=V_{\text {rated }}^{\text {old }}+\left(V_{\text {desired }}-|V|\right)$

This is shown schematically in Fig. 2(c). 
It is to be noted that, restoring the voltage magnitude and frequency to the rated values can also be achieved using a Proportional-Integral (PI) controller. The PI based method will take a much longer time to restore these parameters due to the larger time step of the central controller. However, using the proposed method, the new references can be directly calculated and transmitted to the primary controllers of each DER system.

\section{STUDy CASES AND SIMULATION RESULTS}

To investigate the performance of the proposed MG central controller, the MG system of Fig. 1(a) with 2 DERs (i.e. DER-1 and DER-2) is simulated in PSCAD/EMTDC. The parameters of network, DER converters and filters, coupling inductances, loads and the droop control coefficients are given in the Appendix. Several case studies are built; among which a few are discussed below.

\section{Case-1: MG Autonomous Operation Results}

It is assumed that the MG system is initially in steady-state condition, with a total load demand of approximately $0.41 \mathrm{pu}$ where $1 \mathrm{pu}$ is $6 \mathrm{~kW}$. At $t=0.5 \mathrm{~s}$, this load is increased to $1 \mathrm{pu}$ and at $t=1 \mathrm{~s}$, the network load is decreased to $0.53 \mathrm{pu}$. At $t=$ $1.5 \mathrm{~s}$, the load is further decreased to $0.17 \mathrm{pu}$.

The output active power ratio among DER-1 and 2, is maintained as 1:2, as assigned by the central controller (Fig. 3a) for all load changes. The MG voltage and frequency are within the acceptable limits during all load changes as shown in Fig. 3c-d. Hence, the central controller does not issue any control commands and $V_{\text {rated }}$ is kept as 1 pu while the $f_{\text {rated }}$ is kept as $50 \mathrm{~Hz}$ (Fig. 3e-f).

\section{Case-2: Droop Curve Adjustment Results}

It is assumed that the MG system is initially in steady-state condition, with a total load demand of approximately $0.15 \mathrm{pu}$ where $1 \mathrm{pu}$ is $18 \mathrm{~kW}$. The output power of DERs is maintained as 1:2, as assigned by the central controller (Fig. 4a).

At $t=0.5 \mathrm{~s}$, the load is increased to 0.4 pu (Fig. $4 \mathrm{~b}$ ) and consequently, the network voltage drops to $0.89 \mathrm{pu}$ (Fig. 4c) which is below the acceptable limit of $0.9 \mathrm{pu}$. The MG frequency is still within the acceptable range (Fig. 4d). Since the central controller operates with a larger time step, as discussed before, it modifies $V_{\text {rated }}$ for the system (Fig. 4e) at the first operation time after the load change (i.e. $t=1 \mathrm{~s}$ ). Hence, $V_{\text {rated }}$ is updated to 1.1. It can be seen that, as the central controller increases the voltage reference for the droop control, the network voltage is restored to $1 \mathrm{pu}$.

At $t=1.5 \mathrm{~s}$, another load increase is applied and the system undergoes the same sequence of events as at $t=0.5 \mathrm{~s}$. The central controller increases the voltage reference to recover the voltage drop at $t=2.5 \mathrm{~s}$. In this case, the MG frequency decreases beyond its acceptable limit and hence the central controller updates $f_{\text {rated }}$ to $51.9 \mathrm{~Hz}$ at $t=2.6 \mathrm{~s} \mathrm{(Fig.} \mathrm{4f).} \mathrm{There-}$ fore, the MG frequency is stored back to $50 \mathrm{~Hz} t=2.6 \mathrm{~s}$.

It is to be noted that since the loads were modelled as constant impedance load, after each $V_{\text {rated }}$ increase at $t=1$ and $t=$ $2 \mathrm{~s}$, the load active power consumption is also increased.
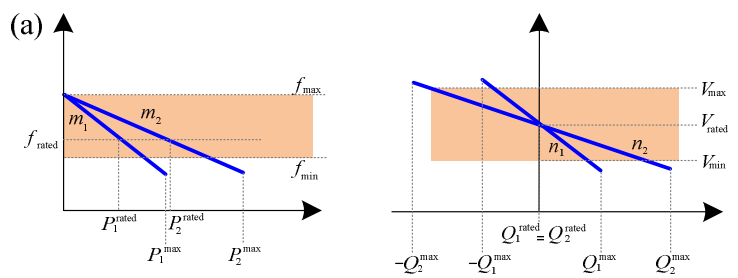

(b)
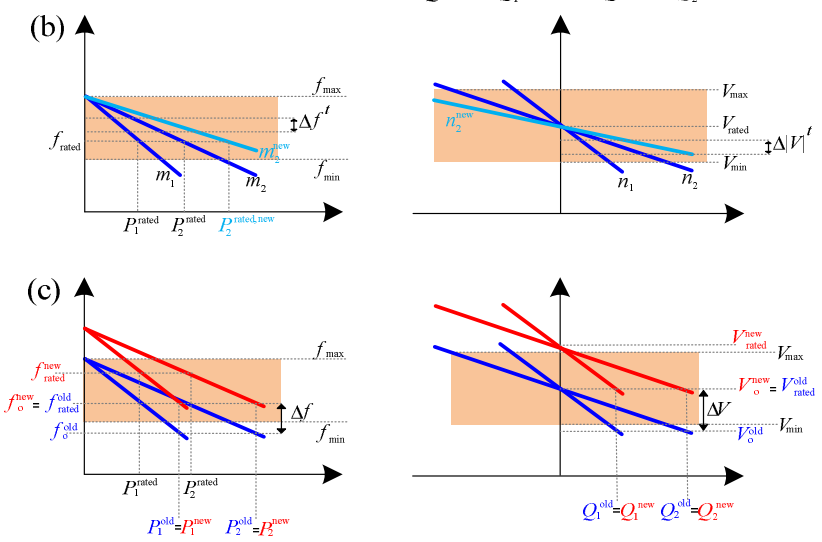

Fig. 2. (a) $P$-f and $Q-V$ droop curves for two DERs, (b) the proposed dynamic droop curve settings adjustment to provide dynamic power ratio change, (c) the proposed droop curve settings adjustment to prevent violation of the voltage and frequency within MG.
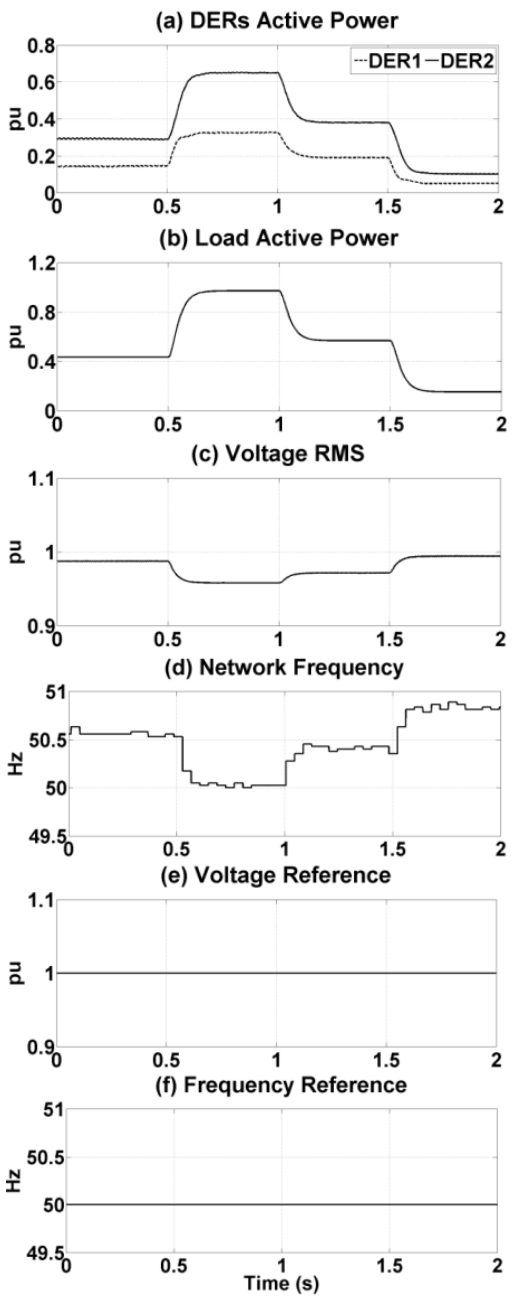

Fig. 3. Simulation results for Case-1. 


\section{Case-3: Dynamic Power Ratio Adjustment Results}

It is assumed that the MG system is in steady-state condition. The two DER systems have the same coupling inductance of $L_{T 1}=L_{T 2}=6.8 \mathrm{mH}$ at their outputs. Initially, the tertiary controller has defined the output active power ratio among DER1:DER2 to be as 1:2 (Fig. 5a). Hence, the droop coefficient ratio of the two DERs is selected as $m_{\text {DER } 1} / m_{\text {DER2 }}=$ $2 / 1$ (Fig. 5b). In addition, it selects $P_{\text {rated } 1} / P_{\text {rated2 }}=2 / 1$. These two ratios are passed to the primary controllers of each DER system.

At $t=0.5 \mathrm{~s}$, the tertiary controller changes the output active power ratio among DER1:DER2 to be as 1:1 and passes to this information to the central controller. Hence, the $P-f$ droop coefficients ratio and rated active power ratio between the two DERs are selected as $m_{\text {DER } 1} / m_{\text {DER2 }}=1 / 1$ and $P_{\text {rated } 1} / P_{\text {rated } 2}=1 / 1$ and then passed to the primary controllers of each DER system.

At $t=1 \mathrm{~s}$, the tertiary controller orders the MG central controller to facilitate the active power output ratio among DER1:DER2 to be as 3:1. Hence, the droop coefficient ratio and rated active power ratio between the two DERs are selected as $m_{\text {DER } 1} / m_{\text {DER } 2}=1 / 3$ and $P_{\text {rated } 1} / P_{\text {rated } 2}=1 / 3$ and then passed to the primary controllers of each DER system.

\section{CONCLUSION}

The microgrid central controller is responsible to keep the voltage magnitude and frequency within the acceptable ranges. This controller runs in a larger time step compared to the primary controllers of the DER systems. Once the voltage magnitude or frequency is violated, it needs to calculate the new references values for the voltage magnitude and frequency and transmit this information to the primary controllers at each DER system. This can be achieved using a PI controller but will have very slow dynamics. However, in this paper, a new method was presented to calculate the new reference value for these parameters directly.

Another functionality of the central controller is to provide dynamically modify the ratio of the droop coefficients and the rated output powers of the DER systems to facilitate the desired output active power ratio among the DER systems. To provide a desired ratio among both the active and reactive powers, a virtual impedance-based technique should be utilized. However, if only the output active power ratio is desired, it can be achieved using the proposed technique.

\section{APPENDIX}

Table I. Technical data of the network parameters of Fig. 1(a).

$\begin{array}{ll}\text { MV Network } & 400 \mathrm{Vrms} \mathrm{L}-\mathrm{L}, 50 \mathrm{~Hz} \\ \text { MV Line Impedance } & R=0.2 \Omega, L=10 \mathrm{mH} \\ \text { VSCs and Filters for } & R_{f}=0.1 \Omega, L_{f}=0.36 \mathrm{mH}, C_{f}=50 \mu \mathrm{F}, V_{d c}=350 \mathrm{~V}, \\ \text { DER systems } & a=1\end{array}$

Table II. Coupling inductances and droop control coefficients for DER-1,2 in simulation case studies 1,2 .

\begin{tabular}{lccc}
\hline DER & $L_{T}[\mathrm{mH}]$ & $m[\mathrm{rad} / \mathrm{kW}]$ & $n[\mathrm{~V} / \mathrm{kVAr}]$ \\
\hline DER-1 & 2.72 & 3.1416 & 18 \\
DER-2 & 1.36 & 1.5708 & 9 \\
\hline
\end{tabular}

(a) DERs Active Power

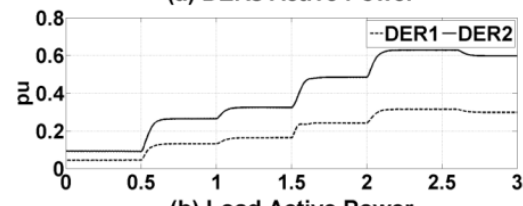

(b) Load Active Power

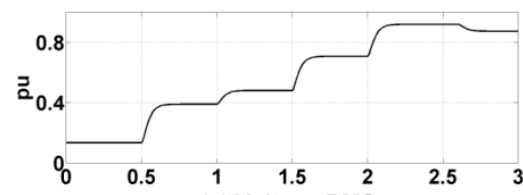

(c) Voltage RMS

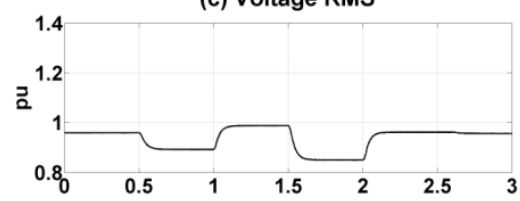

(d) Network Frequency

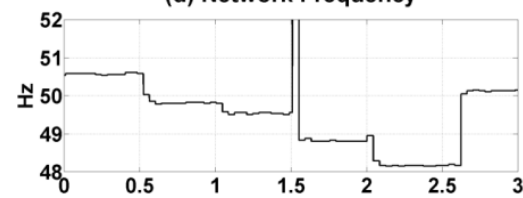

(e) Voltage Reference
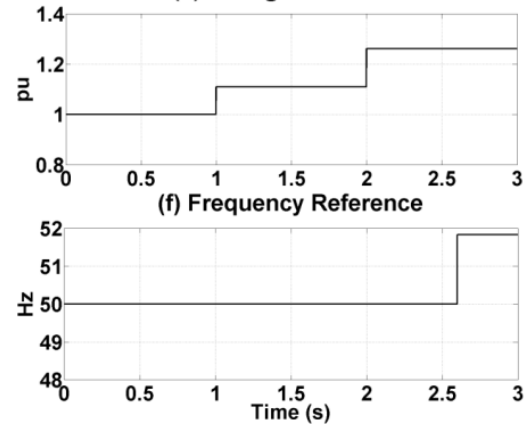

Fig. 4. Simulation results for Case-2.

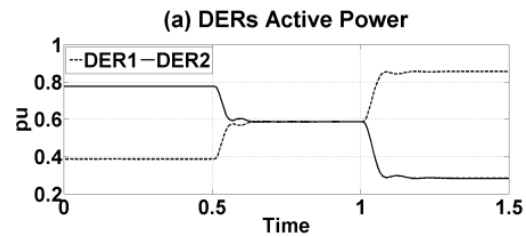

(b) Droop Coefficients Ratio

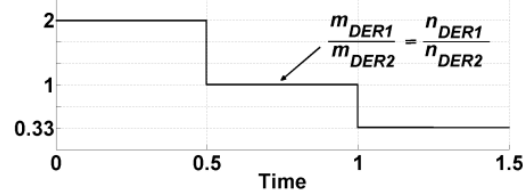

Fig. 5. Simulation results for Case-3.

\section{REFERENCES}

[1]. B Kroposki, C. Pink, R. DeBlasio, H. Thomas, M. Simões and P.K. Sen, "Benefits of Power Electronic Interfaces for Distributed Energy Systems," IEEE Trans. on Energy Conversion, vol. 25, no. 3, pp. 901908, 2010.

[2]. T. Senjyu, T. Nakaji, K. Uezato, and T. Funabashi, "A hybrid power system using alternative energy facilities in isolated island," IEEE Trans. on Energy Conversion., vol. 20, no. 2, pp. 406-414, 2005.

[3]. R.H. Lasseter, "MicroGrids," IEEE Power Engineering Society Winter Meeting, vol. 1, pp. 305-308, 2002. 
[4]. A.G. Madureira and J.A. Pecas Lopes, "Coordinated Voltage Support in Distribution Networks with Distributed Generation and Microgrids," IET Renewable Power Generation, vol. 3, no. 4, pp. 439-454, 2009.

[5]. K. Pandiaraj, P. Taylor, N. Jenkins, and C. Robb, "Distributed Load Control of Autonomous Renewable Energy Systems," IEEE Trans. on Energy Conversion, vol. 16, no. 1, pp. 14-19, 2001.

[6]. R. Majumder, G. Ledwich, A. Ghosh, S. Chakrabarti and F. Zare, "Droop Control of Converter-Interfaced Microsources in Rural Distributed Generation," IEEE Trans. on Power Delivery, vol. 25, no. 4, pp. 2768-2778, 2010.

[7]. S.J. Ahn, J.W. Park, I.Y. Chung, S.I. Moon, S.H. Kang and S.R. Nam, "Power-Sharing Method of Multiple Distributed Generators Considering Control Modes and Configurations of a Microgrid," IEEE Trans. Power Delivery, vol. 25, no. 3, pp. 2007-2016, 2010.

[8]. I.Y. Chung, W. Liu, D.A. Cartes, E.G. Collins and S.I. Moon, "Control Methods of Inverter-Interfaced Distributed Generators in a Microgrid System," IEEE Trans. on Industrial Applications, vol. 46, no. 3, pp. 1078-1088, 2010.

[9]. C.K. Sao and P.W. Lehn, "Control and power management of converter fed microgrids," IEEE Trans. on Power System. vol. 23, no. 3, pp. 1088-1098, 2008.

[10]. F. Shahnia, R. P. S. Chandrasena, S. Rajakaruna and A. Ghosh, "Primary Control Level of Parallel Distributed Energy Resources Converters in System of Multiple Interconnected Autonomous Microgrids within Self-Healing Networks," IET Generation, Transmission.\& Distribution, Vol. 8, no. 2, pp. 203-222, Feb. 2014.
[11]. S.J. Ahn, J.W. Park, I.Y. Chung, S.I. Moon, S.H. Kang and S.R. Nam, "Power-sharing method of multiple distributed generators considering control modes and configurations of a microgrid," IEEE Trans. on Power Delivery, vol. 25, no. 3, pp. 2007-2016, 2010.

[12]. H. Karimi, H. Nikkhajoei and R. Iravani, "Control of an electronically-coupled distributed resource unit subsequent to an islanding event," IEEE Trans. on Power Delivery, vol. 23, no. 1, pp. 493-501, 2008.

[13]. Xiaoxiao Yu, A. M. Khambadkone, Huanhuan Wang, and S. Ter-ence, "Control of parallel-connected power converters for low-voltage microgrid-part I: A hybrid control architecture," IEEE Trans. on Power Electronics, vol. 25, no. 12, pp. 2962-2970, 2010.

[14]. M. Hamzeh, H. Karimi and H. Mokhtari, "A new control strategy for a multi-bus mv microgrid under unbalanced conditions," IEEE Trans. on Power System, vol. 27, no. 4, pp. 2225-2232, Nov. 2012.

[15]. F. Shahnia, R. Majumder, A. Ghosh, G. Ledwich and F. Zare, "Operation and control of a hybrid microgrid containing unbalanced and nonlinear loads," Electric Power System Research, vol. 80, no. 8, pp. 954965, Aug. 2010.

[16]. J. M. Guerrero, L. Garcia de Vicuna, J. Matas, M. Castilla, and J. Miret, "Output Impedance Design of Parallel-Connected UPS Inverters With Wireless Load-Sharing Control," IEEE Trans. on Industrial Electronics, vol. 52, no. 4, pp. 1126-1135, Aug. 2005.

[17]. M.J. Sanjari and G.B Gharehpetian, 'Small signal stability based fuzzy potential function proposal for secondary frequency and voltage control of islanded microgrid', Electric Power Components Systems, 2013, vol 41, no.5, pp. 485-499

[18]. F. Katiraei, R. Iravani, N. Hatziargyriou, and A. Dimeas, "Microgrids management," IEEE Power Energy Magazine. vol. 6, no. 3, pp. 54-65, 2008. 